This item was submitted to Loughborough's Research Repository by the author.

Items in Figshare are protected by copyright, with all rights reserved, unless otherwise indicated.

\title{
Determining the solution space for a coordinated whole body movement in a noisy environment: application to the upstart in gymnastics
}

PLEASE CITE THE PUBLISHED VERSION

http://dx.doi.org/10.1123/jab.2013-0282

\section{PUBLISHER}

(c) Human Kinetics, Inc.

\section{VERSION}

AM (Accepted Manuscript)

\section{PUBLISHER STATEMENT}

This work is made available according to the conditions of the Creative Commons Attribution-NonCommercialNoDerivatives 4.0 International (CC BY-NC-ND 4.0) licence. Full details of this licence are available at: https://creativecommons.org/licenses/by-nc-nd/4.0/

\section{LICENCE}

CC BY-NC-ND 4.0

\section{REPOSITORY RECORD}

Hiley, Michael J., and Maurice R. Yeadon. 2019. "Determining the Solution Space for a Coordinated Whole Body Movement in a Noisy Environment: Application to the Upstart in Gymnastics". figshare. https://hdl.handle.net/2134/16166. 


\title{
Determining the solution space for a co-ordinated whole body movement in a noisy environment: application to the upstart in gymnastics
}

\author{
Michael J. Hiley and Maurice R. Yeadon
}

School of Sport, Exercise \& Health Sciences, Loughborough University, Loughborough, UK

\begin{abstract}
The upstart is a fundamental skill in gymnastics, requiring whole body co-ordination in order to transfer the gymnast from a swing beneath the bar to a support position above the bar. The aim of this study was to determine the solution space within which a gymnast could successfully perform an upstart. A previous study had shown that the underlying control strategy for the upstart could be accounted for by maximising the likelihood of success whilst operating in a noisy environment. ${ }^{1}$ In the current study data were collected on a senior gymnast and a computer simulation model of a gymnast and bar was used to determine the solution space for maximising success whilst operating in a noisy environment. The effects of timing important actions, gymnast strength and movement execution noise on the success of the upstart were then systematically determined. The solution space for the senior gymnast was relatively large. Decreasing strength and increasing movement execution noise reduced the size of the solution space. A weaker gymnast would have to use a different technique to that used by the senior gymnast in order to produce an acceptable success rate.
\end{abstract}

Keywords: simulation, optimisation criterion, movement variability, gymnastics

\section{INTRODUCTION}

The upstart is a fundamental skill in gymnastics which requires whole body coordination in order to transfer the gymnast from a swing beneath the bar to a support position above the bar (Figure 1). The skill is used by both elite and beginner gymnasts in men's and women's artistic gymnastics on the high bar, parallel bars and the uneven bars. The upstart comprises a swinging phase (Figure $1 \mathrm{~A}-\mathrm{B}$ ) and a "kip" phase (Figure 1 C-D). The upstart should be performed with straight arms and straight legs in order to receive no deductions from the judges. ${ }^{2}$

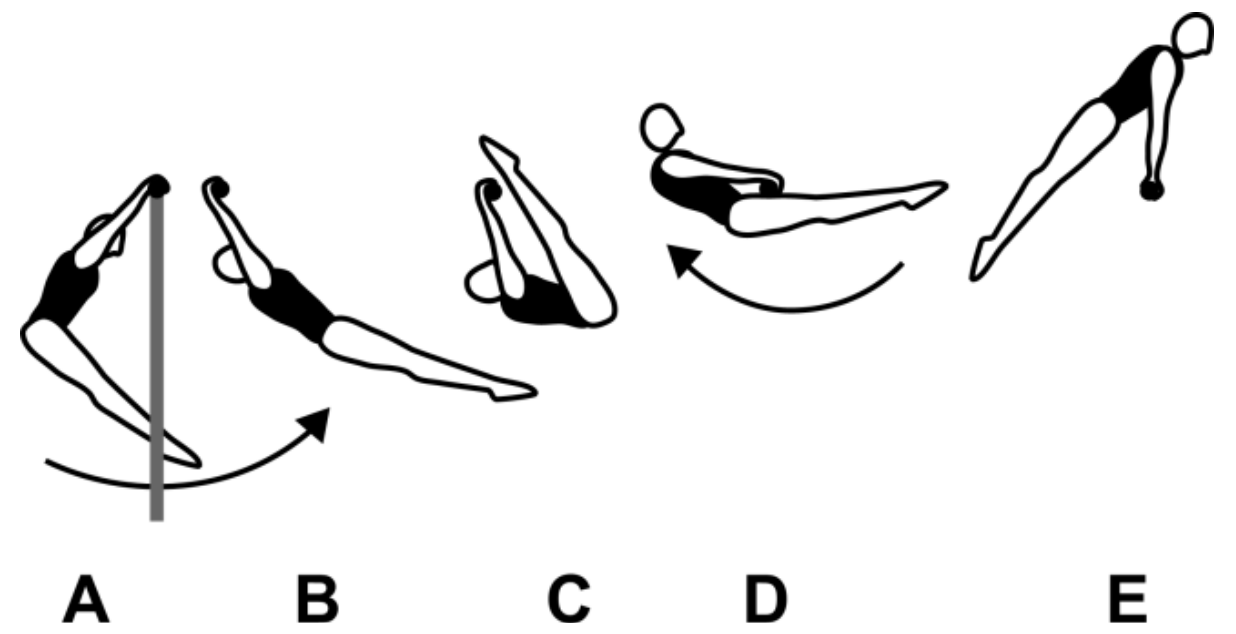

Figure 1. The upstart (adapted from the FIG Code of Points, 2013). 
Recent research has focused on determining the control strategy used in the upstart through computer simulation modelling. The technique (joint angle time histories of the hip and shoulder) for the upstart has previously been optimised using various criteria based on minimising angle jerk, joint torque change and effort. ${ }^{3}$ Only when the joint angle time histories were forced through arbitrary via-points (at which the shoulder and hip angles were required to match the recorded gymnast angles) did the model reproduce the movements of the gymnasts. ${ }^{3}$ Since the via-points were placed arbitrarily it could not be concluded that any of the criteria explained the technique used by the gymnasts. Therefore, there must have been some other criterion that explains the gymnasts' technique or some important aspect of human movement that had been overlooked.

The study of Yamasaki et al. ${ }^{3}$ has since been repeated ${ }^{1}$, but with an extra criterion based on maximising success at the task whilst operating in a noisy environment. ${ }^{4}$ The optimisation added noise to the joint angle time histories based on measures of human movement variability ${ }^{5}$ which were used to represent movement execution noise resulting from the motor system. ${ }^{6}$ For each time history produced by the optimisation algorithm, 500 randomly perturbed simulations were run with the objective function score being based on how many simulations resulted in a successful upstart. This optimisation criterion produced solutions with techniques closer to the gymnast performance than any of the other criteria. The maximising success criterion has been shown to characterise gymnasts' technique in other swinging skills. ${ }^{7}$ In other words, gymnasts' technique is characterised by maximising success in a noisy environment.

If there are many constraints as in the upstart (e.g. strength limits, levels of noise within the system and configurational end point requirements), the solution space for success may be small. An understanding of the solution space, in terms of timing key actions, strength limits and the amount of noise in movement execution ${ }^{6}$ could provide insight into what causes a gymnast to fail at the task when acquiring this complex skill.

The aim of the present study is to determine the solution space for the upstart and to investigate how it is influenced by the timing of key actions, gymnast strength and the amount of noise in the gymnast's movement execution. It is hypothesised that the solution space for an elite senior gymnast will be relatively large compared to a novice gymnast since the constraints imposed by strength limits and levels of execution noise will be less limiting for the elite gymnast. Additionally reductions in maximum strength and increases in execution noise may be expected to lead to a smaller solution space.

\section{METHODS}

\section{Data collection}

One male gymnast (age 24 years, mass $70 \mathrm{~kg}$, height $1.73 \mathrm{~m}$ ) who competed internationally gave informed consent to participate in the study in accordance with the university Ethical Advisory Committee requirements. The gymnast performed an upstart on a low bar (height $1.55 \mathrm{~m}$ ) and was recorded using 17 Vicon MX13 cameras operating at $300 \mathrm{~Hz}$. Spherical reflective markers, $25 \mathrm{~mm}$ in diameter, were attached to the lateral side of the wrist, elbow, shoulder, hip, knee and ankle joint centres and toes on the left side of the body. Offset measurements from each marker centre to the adjacent joint centre were recorded for subsequent location of the joint centres. Additional markers were attached to each side of the gymnast's 
head (above the ear) and to the centre of the bar. Prior to data collection a volume centred on the bar was wand calibrated using the motion analysis system.

Three-dimensional marker coordinates were reconstructed and joint centres were determined using the measured offsets. Joint angles were calculated from the joint centre coordinates and fitted with quintic splines ${ }^{8}$ for use with the simulation model. The arm angle was used as the orientation angle and was defined as the angle made by the line joining the wrist to the shoulder and the upward vertical (Figure 2). Segmental inertia parameters were determined from anthropometric measurements taken on the gymnast using an inertia model. ${ }^{9}$

\section{Simulation model}

A four segment planar model of a gymnast comprising arm, torso, thigh and lower leg segments was used to simulate the movement around the bar. ${ }^{10}$ The bar and the gymnast's shoulder structure were modelled as damped linear springs (Figure 2).

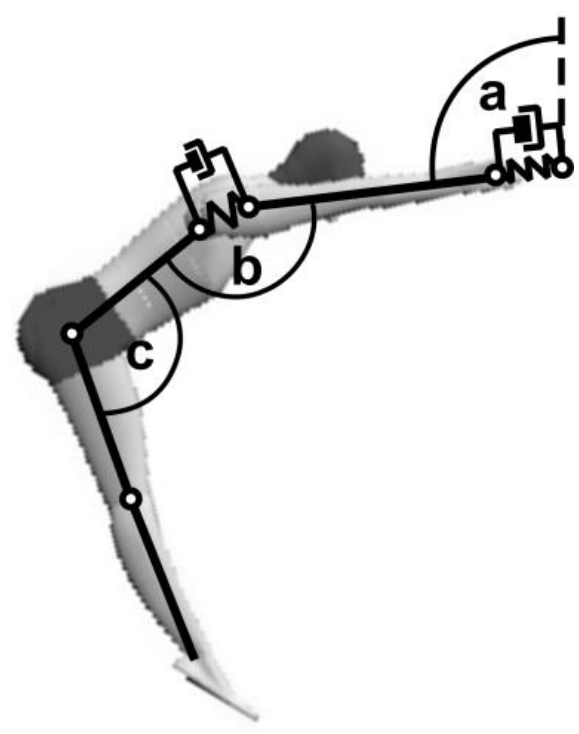

Figure 2. The four segment gymnast - bar simulation model with damped springs representing bar and shoulder elasticity together with (a) orientation angle of the arm and joint angles at (b) shoulder and (c) hip.

Input to the simulation model comprised the segmental inertia parameters, the stiffness and damping coefficients of the bar and shoulder springs, the initial displacement and velocity of the bar, the initial angular velocity of the arm, the initial orientation of the arm and the joint angle time histories of the shoulder, hip and knee in the form of quintic splines. Output from the model included the time histories of the horizontal and vertical bar displacements and the arm angle. To obtain model parameters which could not be calculated directly (e.g. spring stiffness and damping coefficients) a matching procedure was carried out as described previously. ${ }^{11} \mathrm{~A}$ simulation was run with the matched parameters to assess the closeness of fit to the recorded data. The root mean squared differences between the matching simulation and the recorded performance for the arm angle and bar displacements were $1^{\circ}$ and $0.005 \mathrm{~m}$ (Figure 4). 


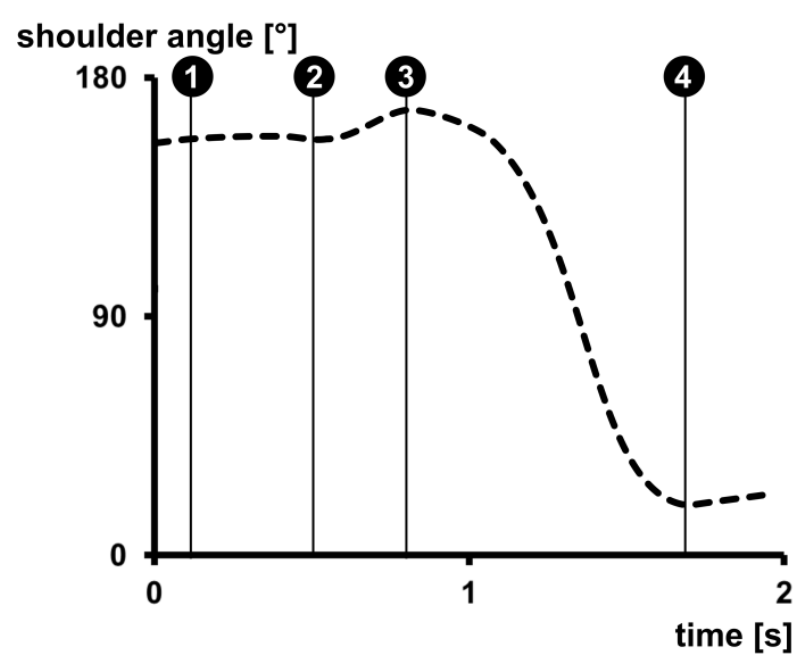

(a)

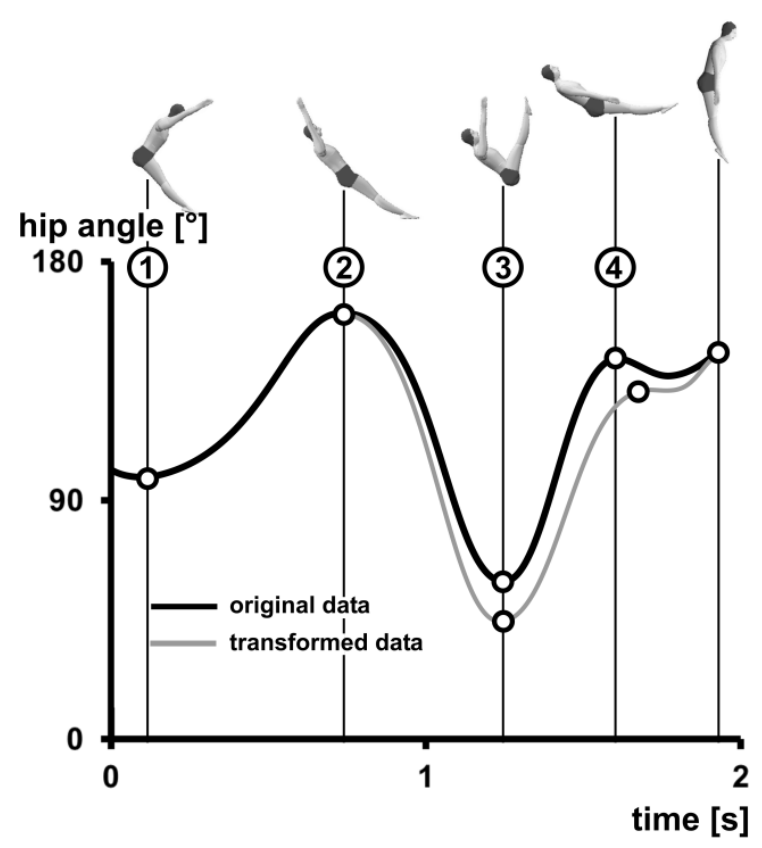

(b)

Figure 3. The joint angle history at (a) the shoulder, (b) the hip during the upstart with the turning points indicated and (c) the method of transforming the angle data between two turning points.

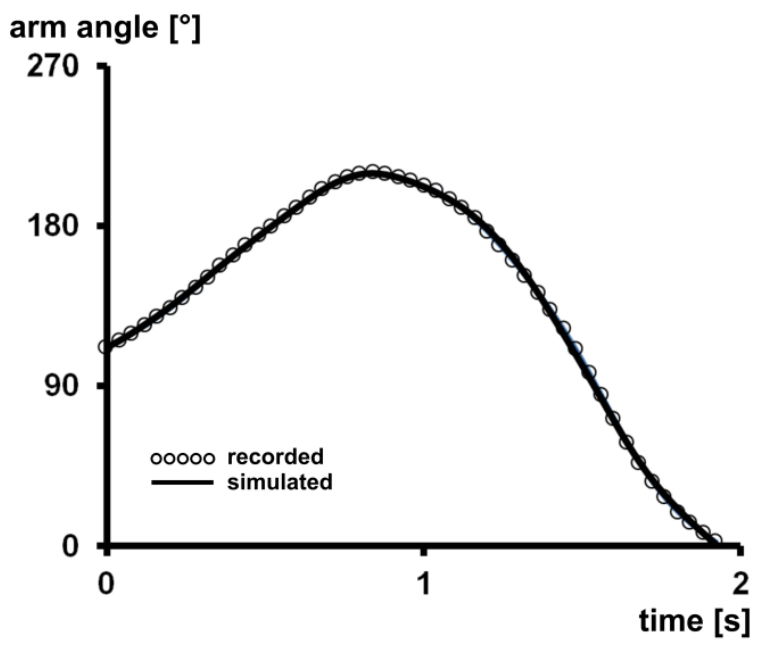

(a)

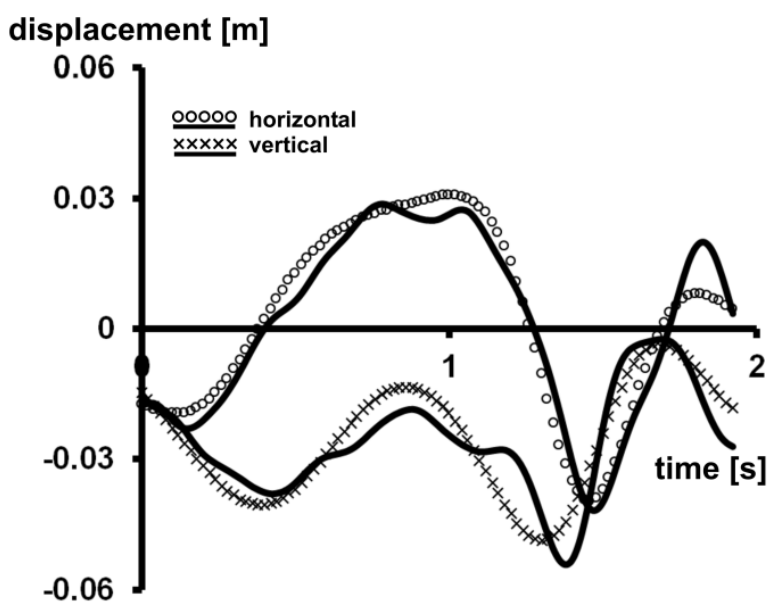

(b)

Figure 4. Matching simulation (solid lines) compared with recorded performances (circles and crosses) for: (a) arm angle and (b) bar displacements.

\section{Optimisation}

The simulation model was incorporated within a parallelised genetic optimisation algorithm ${ }^{12,13}$ which manipulated parameters defining the joint angle time histories at the shoulder and hip. The parameters comprised the time and angle at each of four turning points in the shoulder and hip time histories (Figure 3, i.e. 16 parameters). A data set spanning each joint angle time history obtained from the video analysis was transformed between each turning point in both the time and angle dimensions (Figure 3b). An interpolating quintic spline was then fit to the new 
data set to allow the calculation of first and second derivative data required to drive the simulation model. Throughout the simulations the legs were constrained to stay straight in keeping with good form.

Two optimisations were carried out to determine the technique that maximised success for the upstart. The first had the strength constraints set to the maxima of the gymnast and the second had the strength set to $75 \%$ of the gymnast's maximum values. The second optimisation was used to represent a gymnast who was less strong (e.g. a younger or less well conditioned gymnast) and demonstrate how technique is affected by strength limits. The strength was constrained using joint torque measurements determined from an isovelocity dynamometer for the gymnast and by fitting a function which expressed maximum voluntary torque in terms of joint angle and angular velocity. ${ }^{14,15}$

All simulations performed within the optimisations started with an arm angle of $110^{\circ}$ and the initial conditions from the recorded performance and ended once the arm angle reached the vertical support position above the bar (Figure 3b). Since a solution close to the gymnast's performance was expected for the first optimisation, the bounds for each parameter were initially set to either $\pm 5^{\circ}$ or to $\pm 0.05 \mathrm{~s}$ of the average angle or time values. If an optimal solution reached any of the bounds the optimisation was repeated using the current solution as a starting point with a new set of bounds as described above. This was repeated until the optimal solution achieved $100 \%$ success and lay within the bounds. The process was repeated for the second optimisation with reduced strength, but since a change in technique was expected, the initial bounds were increased to $\pm 30^{\circ}$ and $\pm 0.30 \mathrm{~s}$. In both optimisations the recommended optimisation algorithm tuning was used. ${ }^{12}$

The optimisation criterion was based on maximising the number of successful upstarts despite operating in a "noisy" environment. For each set of joint angle time history parameters produced by the genetic optimisation algorithm, an unperturbed simulation was run together with 499 randomly perturbed simulations based on the same set of parameters, where perturbations were added to the time and angle parameters of the joint angle histories with a specified standard deviation. The standard deviations were determined from repeated trials of 10 accelerated giant circles by the gymnast ${ }^{16}$ with the average standard deviations from the turning points at the shoulder and hip being used (12 $\mathrm{ms}$ and $\left.1.5^{\circ}\right)$. The perturbations were added using a normally distributed random number generator. ${ }^{5}$ The perturbations were used to represent the noise in the execution of the movements. ${ }^{6}$ For this reason the measures were taken from giant circles, where it appears that the gymnast was attempting to minimise the variation in technique ${ }^{16}$. In tasks that require precise timing it has been shown that kinematic variability is minimised in the important actions $^{16,17}$. If the gymnast has attempted to minimise kinematic variability then the measured values at these instants will therefore have a large component of noise ${ }^{18}$. Simulations were given a score of 1 for a successful upstart and 0 for an unsuccessful upstart. A successful simulation was one in which the model reached the correct final orientation with an appropriate configuration (with the shoulder and hip angles within $10^{\circ}$ of the recorded performance to allow for the addition of noise to the angle parameters). Exceeding the joint torque limits or the legs passing through the bar constituted an unsuccessful simulation. The unperturbed simulation was deemed unsuccessful if the duration was more than $10 \%$ different from the recorded performance. This penalty was used to encourage a similar duration to the recorded performance. 


\section{Determining the solution space}

In order to investigate the solution space for the upstart, the time parameters defining the hip and shoulder joint angle time histories of the two optimal solutions (full strength and reduced strength) were systematically varied: specifically, the time at which the gymnast reached maximum hip flexion (Figure 3, (3) and the end time of the subsequent hip extension (Figure 3, (4)). In effect this varied the time at which the feet were brought to the bar and the duration of the kip (Figure 1, C-D). Since the end of the shoulder extension appeared to be co-ordinated with the end of the kip in the optimal solutions (Figure $3, \boldsymbol{4})$ the end time of the shoulder extension was varied with the end time of the hip extension (i.e. to maintain the co-ordination between hip and shoulder). Both parameters were varied by -0.50 to $0.50 \mathrm{~s}$ in steps of $0.01 \mathrm{~s}$ about the optimal values. For each combination 500 randomly perturbed simulations were run to assess how successful the combination was (using the same criteria for success as the optimisation). The effects of a $25 \%$ reduction in strength and a $25 \%$ increase in the movement execution noise were determined for the two optimisations. Contour graphs of success against the two time parameters were plotted. This process was repeated for the $75 \%$ strength optimal solution.

\section{RESULTS}

Varying the maximum strength of the simulation model had a marked effect on the solution. In the first optimisation, with full strength, a solution that resulted in $100 \%$ success was found close to the gymnast's technique (Figure 5). However, when the strength was reduced by $25 \%$ and the technique re-optimised (second optimisation), the solution that produced $100 \%$ success diverged from the recorded performance (Figure 5). The reduced strength optimum technique had less hip extension at the front of the swing, less hip flexion as the feet approached the bar, and a lower rate of hip flexion and extension compared to the recorded performance (Figure 5). However, the shoulder extension still appeared to be co-ordinated with the end of the kip (Figure 5).

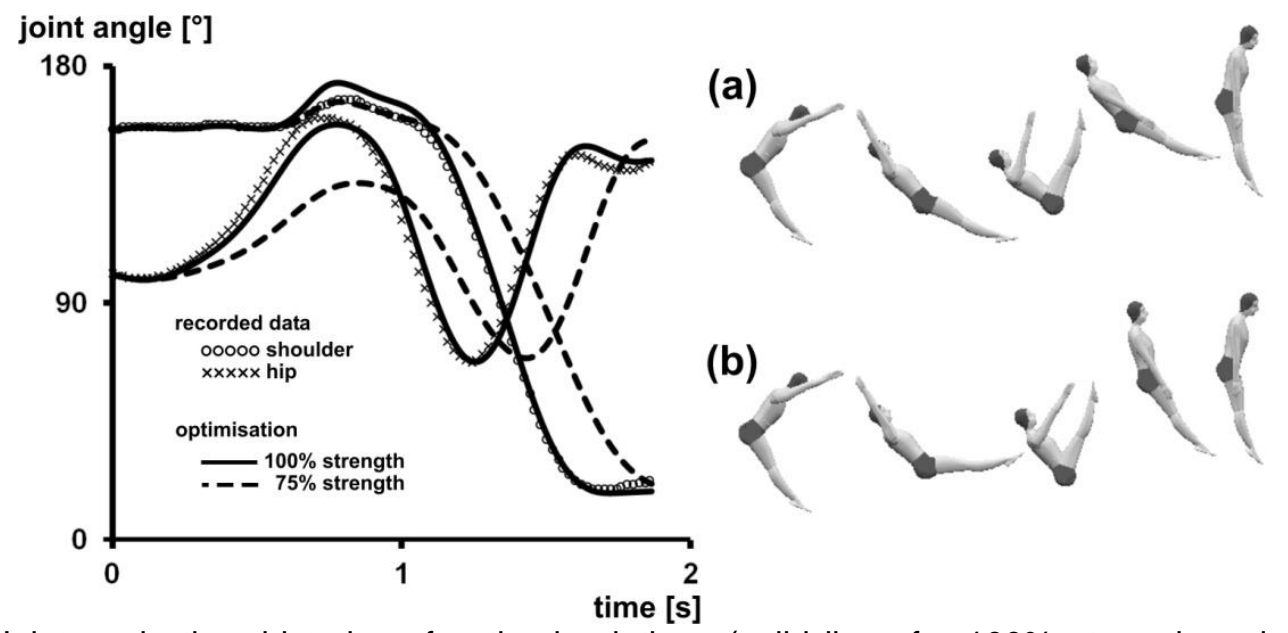

Figure 5. Joint angle time histories of optimal solutions (solid lines for $100 \%$ strength and dashed lines for $75 \%$ strength solutions) compared with the recorded histories (circles and crosses) along with graphics sequences of the (a) $100 \%$ strength and (b) $75 \%$ strength solutions.

For the first optimisation the solution space was relatively large in the two timing variables (Figure 6a) although, the gymnast's technique was not centred in the region defining $99-100 \%$ success (as the optimal technique was constrained to stay close to the recorded performance). When the movement execution noise added to the joint 
angle time histories was increased by $25 \%$ the solution space defining $99-100 \%$ success became smaller (Figure 6b). A more notable reduction in successful solution space was obtained when the strength of the gymnast was reduced by $25 \%$ (Figure 6c), and when the effect of increased movement execution noise and decreased strength were combined (Figure 6d). For the last two cases there was no longer a region defined by $90-100 \%$ success (Figure 6, $c$ and d).

For the second optimisation, which started with a $25 \%$ reduction in strength, the hip timing solution space was smaller than for the first optimisation (Figure 7a). When the movement execution noise was increased by $25 \%$ the region defined by $99-100 \%$ success disappeared (Figure $7 \mathrm{~b}$ ). When the strength of the model was reduced by a further $25 \%$ the level of success fell to below $10 \%$.

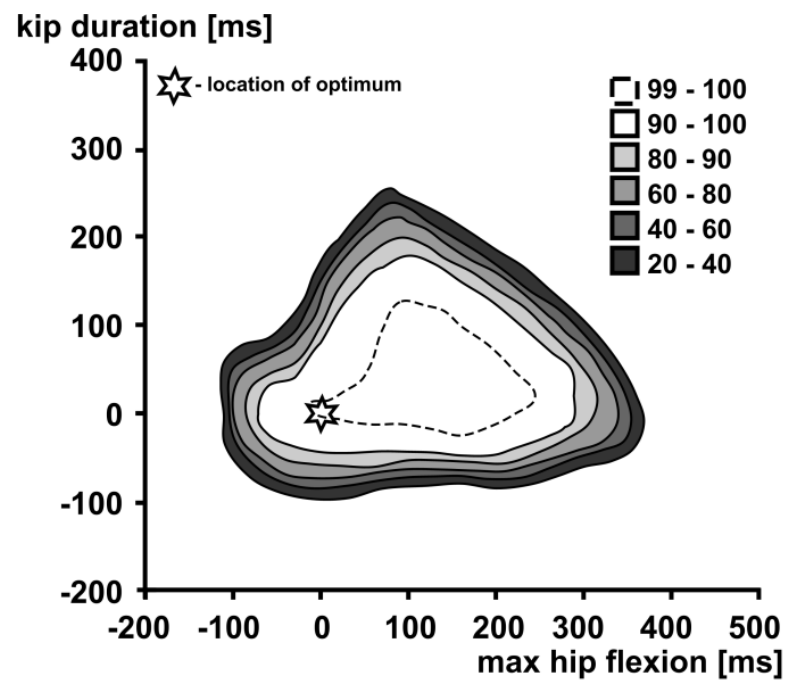

(a)

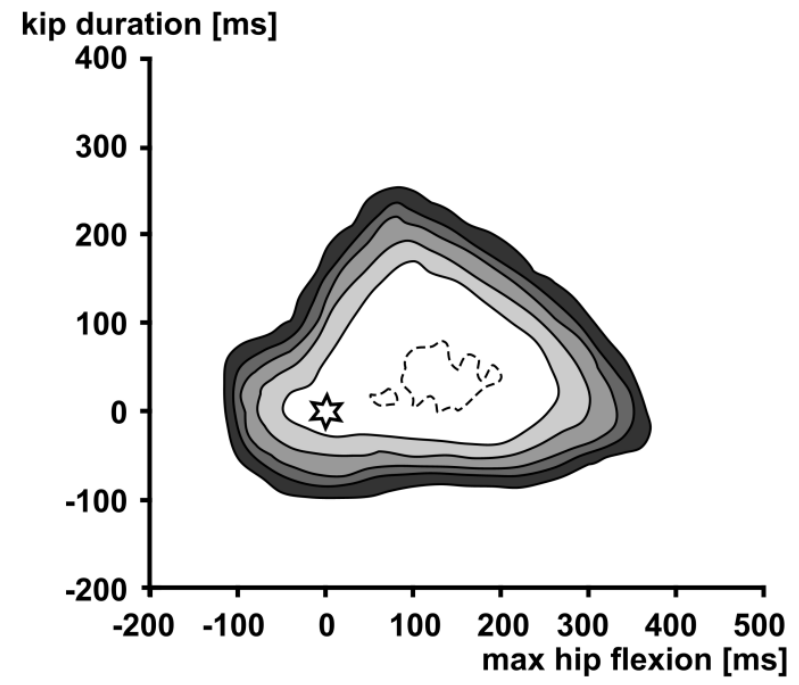

(b)

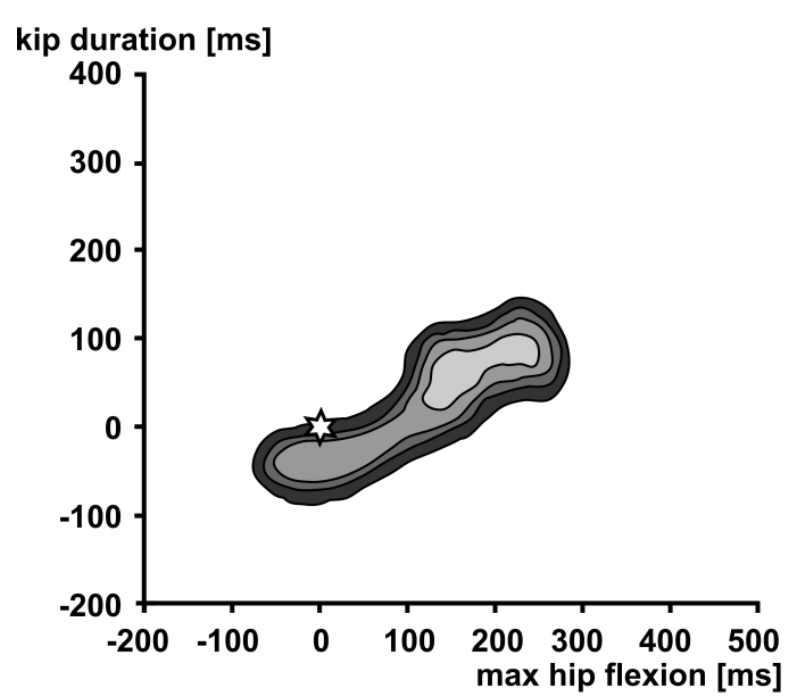

(c)

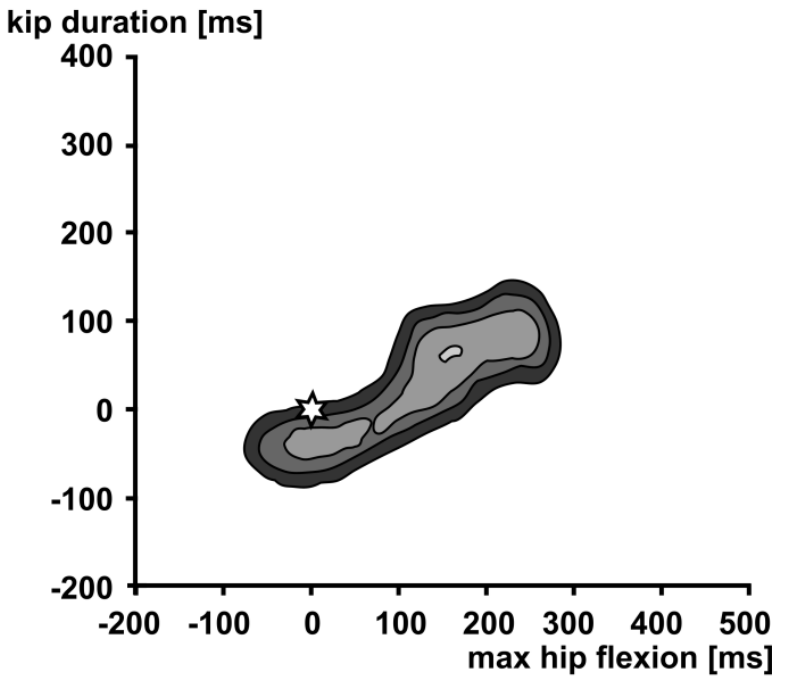

(d)

Figure 6. Contour plots of percentage success of the full strength optimal solution against changes in time at peak hip flexion (feet to the bar) and duration of the subsequent hip extension (kip) for: (a) optimal solution, (b) kinematic movement variability increased by $25 \%$, (c) strength reduced by $25 \%$ and (d) movement variability increased by $25 \%$ combined with strength reduced by $25 \%$. 


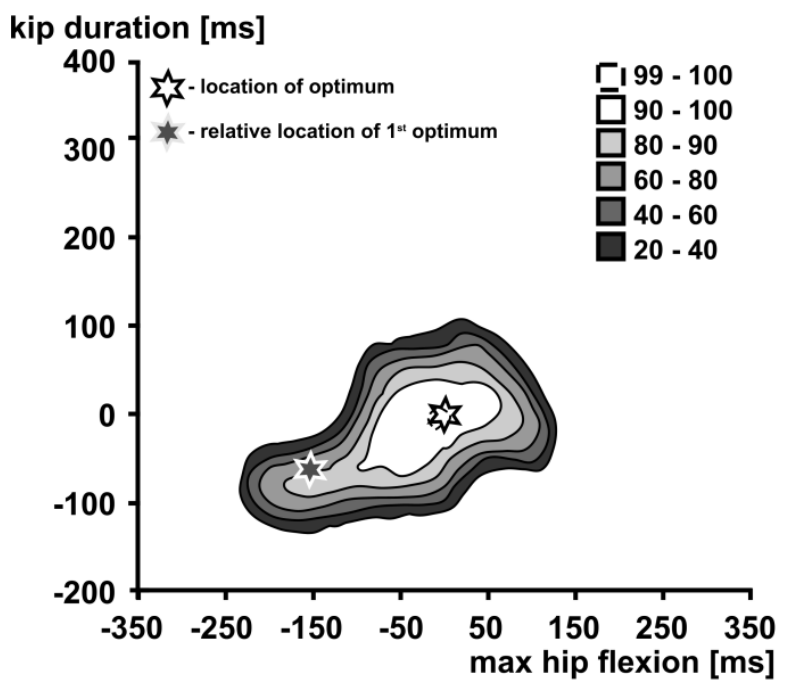

(a)

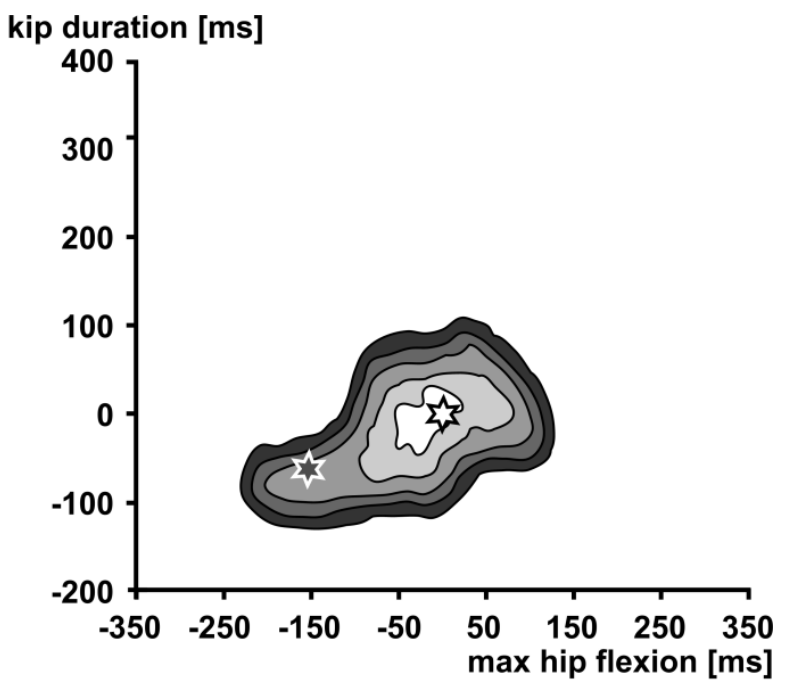

(b)

Figure 7. Contour plots of percentage success of the $75 \%$ strength optimal solution against changes in time at peak hip flexion (feet to the bar) and duration of the subsequent hip extension (kip) for: (a) optimal solution and (b) kinematic movement variability increased by $25 \%$.

\section{DISCUSSION}

The aim of the study was to determine the solution space for the upstart and to investigate how it is affected by the timing of key actions, gymnast strength and the amount of execution noise in the gymnast's movements. Understanding how the various factors influence the solution space will provide insight into what causes a gymnast to fail at the task and help explain why gymnasts have difficulty acquiring this fundamental skill.

When the upstart was optimised to maximise success, a solution which produced $100 \%$ success was found close to the recorded performance (Figure 5), suggesting that the gymnast's technique was characterised by this criterion. When the upstart was re-optimised with the maximum strength limit reduced by $25 \%$, the optimal solution, despite producing $100 \%$ success, diverged from the recorded performance (Figure 5). The major change in technique was a decrease in the range and rate of the hip flexion (Figure $3 b$, from (2) to (3) prior to the kip. In a previous study on the upstart ${ }^{19}$, cited by $\mathrm{Hay}^{20}$, the timing of the hip flexion in relation to the whole body rotation was considered to be a "crucial element" of the skill. In the reduced strength optimal solution, the end of the hip flexion was approximately 150 ms later and the duration of the kip (hip extension) was $65 \mathrm{~ms}$ longer compared to the full strength solution. It would appear that the reduced strength solution has moved towards the central region of $90-100 \%$ success in the full strength solution space (Figure 6a).

It is important to identify those aspects of technique that differ between gymnasts of varying strength, since it may not be possible for younger or less strong gymnasts to achieve the technique desired by the coach. Gymnasts are usually encouraged to achieve full hip extension $\left(180^{\circ}\right)$ at the front of the swing (Figure 1B and Figure 3(2), as failure to do so may result in a points deduction ${ }^{2}$. This may not be optimal for all gymnasts, as it increases the demands placed on the subsequent hip flexion. If the coach decides it is important for the younger/weaker gymnast to use the same technique as the senior gymnast (i.e. that is the end goal), then the coach 
should expect the gymnast to be less successful until such time as he/she becomes sufficiently strong. It may also be necessary for the coach to consider the effect of fatigue on strength and hence solution space. A gymnast may be able to perform the upstart with the desired full extension at the front of the swing during a one-off attempt, but may struggle when performing the skill in a routine. Drills that help the gymnast to learn the timing of the kipping action with the swing, such as the drop upstart $^{21}$, supported upstarts from a piked hang ${ }^{21,22}$, and upstarts where the gymnast does not fully extend at the front of the swing may therefore be beneficial in the early stages of learning the upstart.

For the first optimal solution (close to the recorded performance) it appears that the gymnast has sufficient strength that the solution space is relatively large (Figure 6). In other words success can be achieved within a wide range of timing parameters and so there is the possibility of large kinematic variability. When a gymnast is less strong the possible solutions are reduced compared to the stronger gymnast (Figure $6 \mathrm{a}$ and $7 \mathrm{a})$. As a consequence the timing of the important actions becomes more critical.

In the important actions performed within accelerated giant circles prior to a dismount (hip extension and flexion), it has been shown that as the skill level of the gymnasts increased the amount of movement variability decreased. ${ }^{16}$ In other words, the more elite gymnasts were able to time their actions more precisely. Since the final extension and flexion of the hips had a direct influence on the success of the subsequent dismount, the gymnasts minimised the amount of movement variability at these important times.

If the experiment were to be repeated for the upstart, with senior and junior gymnasts, we might expect to find a ' $U$ ' shaped relationship between kinematic movement variability and skill level, as found for triple jumpers ${ }^{23}$, rather than the decreasing relationship proposed by Newell et al. ${ }^{24}$ and found by others. ${ }^{18}$ During the initial stages of learning, increased levels of variability may be observed as participants "discover" new strategies and techniques. ${ }^{24}$ Then as a successful technique is adopted, movement variability has been shown to decrease with practice. $^{25}$ Since timing the actions is more critical to a successful upstart for a less strong gymnast, there might be less kinematic movement variability as the gymnast attempts to stay within a smaller solution space. Then as the gymnast develops and becomes stronger, the solution space expands and timing of the hip actions becomes less critical, more kinematic variability may be observed. The increased kinematic variability seen in expert performance may also be associated with better error detection and feedback correction. ${ }^{26}$ Future research may wish to look at the detection of errors and the form of the corresponding feedback corrections made during such movements, particularly in situations where the initial conditions are not so well defined as in the present study (e.g. variation in the amplitude of the swing due to the preceding skill).

Cohen and Sternad ${ }^{18}$ presented the solution space for a targeted throwing task, where the measured velocity and angle of projection were plotted against the resulting calculated distance from the target. The movement variability from repeated trials was decomposed into components of tolerance (how far the movements were from the successful solution space), noise (what the optimal level of noise was) and covariation (variation that was compensated for by redundancy) in relation to the task outcome. The study also looked at how these three costs to performance changed with practice. The solution space and decompositions were derived from recorded data rather than from systematically varying the solution, 
although, some comparisons can be made with the present study. Increasing the level of noise in the upstart had the effect of reducing the solution space (Figures 6, a to $b, c$ to $d$ and Figure 7 , a to b). It has been demonstrated that, although noise cannot be removed, its effect on the outcome may be reduced with practice. ${ }^{18}$ Thus as gymnasts learn new skills they will develop techniques that are better able to cope with the level of noise resulting from the motor system. ${ }^{1,4,27}$ An indication of the tolerance and covariation of the optimal upstart technique can be inferred from the regions of the contour plots associated with an acceptable success rate (Figure 6 and 7). Typically an elite gymnast would expect to have success rates in excess of $90 \%$ before including a skill within a competitive routine. Since it has been shown that all three costs (tolerance, noise and covariation) can decrease with practice ${ }^{18}$, it would be interesting to follow a group learning the upstart to see whether this result is obtained for a whole body co-ordinated task, and to see how technique changes with proficiency and physical development.

In the early stages of learning the upstart, the main obstacles to success are associated with adopting a non-optimal technique and a lack of strength. If the gymnast is not sufficiently strong it may be difficult to find a technique with a suitably large solution space, leading to frequent failure at the task. This difficulty may be compounded by the Code of Points ${ }^{2}$, which requires the gymnast to show a fully extended hip angle at the front of the swing. Moving from a fully extended hip angle will require large joint torques which may be problematic for a weaker gymnast. Once a successful technique has been established, continued practice will lead to a refining of technique and a reduction in errors due to planning and execution. ${ }^{6,26}$ As the relative strength of the gymnast increases so will the solution space, leading to higher success rates.

\section{ACKNOWLEDGEMENT}

The authors would like to acknowledge the contribution of Robert Payne and Lydia Philpott in the collection of the upstart data.

\section{REFERENCES}

1. Hiley MJ, Yeadon MR. Investigating optimal technique in a noisy environment: application to the upstart on uneven bars, Hum Movement Sci. 2013; 32:181191.

2. Fédération Internationale de Gymnastique. Code of Points. Moutier, Switzerland: F.I.G; 2013.

3. Yamasaki T, Gotoh K, Xin X. Optimality of a kip performance on the high bar: An example of skilled goal-directed whole body movement. Hum Movement Sci. 2010; 29:464-482.

4. Wolpert DM. Probabilistic models in human sensorimotor control. Hum Movement Sci. 2007; 26:551-524.

5. Hiley MJ, Yeadon MR. Achieving consistent performance in a complex whole body movement: the Tkatchev on high bar. Hum Movement Sci. 2012; 31:834843.

6. van Beers RJ, Haggard P, Wolpert DM. The role of execution noise in movement variability. J Neurophysiol. 2004; 91:1050-1063. 
7. Hiley MJ, Yeadon MR. Investigating optimal technique in a noisy environment: application to the double layout somersault dismount on high bar, $J$ Biomech. submitted May 2013.

8. Wood GA, Jennings LS. On the use of spline functions for data smoothing. $J$ Biomech. 1979; 12:477-479.

9. Yeadon MR. The simulation of aerial movement - II. A mathematical inertia model of the human body. J Biomech. 1990; 23:67-74

10. Hiley MJ, Yeadon MR. Optimum technique for generating angular momentum in accelerated backward giant circles prior to a dismount. J Appl Biomech. 2003; 19:119-130.

11. Hiley MJ, Yeadon MR. Optimisation of backward giant circle technique on the asymmetric bars. J Appl Biomech. 2007; 23:301-309.

12. Carroll DL. FORTRAN genetic algorithm driver. 2001;Downloaded from: http://cuaerospace.com/carroll.ga.html

13. van Soest AJ, Casius RLJ. The merits of a parallel genetic algorithm in solving hard optimiszation problems. J Biomed Eng. 2003; 125:141-146.

14. Forrester SE, Yeadon MR, King MA, Pain MTG. Comparing different approaches for determining joint torque parameters from isovelocity dynamometer measurements. J Biomech. 2011; 44:955-961.

15. King MA, Kong PW, Yeadon MR. Determining effective subject-specific strength levels for forward dives using computer simulation of recorded performances. $J$ Biomech. 2009; 42:2672-2677.

16. Hiley MJ, Zuevsky VV, Yeadon MR. Is skilled technique characterised by high or low variability? - An analysis of high bar giant circles, Hum Movement Sci. 2013;32:171-180.

17. Broderick MP, Newell KM. Coordination patterns in ball bouncing as a function of skill. J Motor Behav. 1999; 31:165-188.

18. Cohen RG, Sternad D. Variability in motor learning : relocating, channeling and reducing noise. Exp Brain Res. 2009; 193:69-83.

19. Bevan R, Corser T. Biomechanical study of gymnastic movements. The Gymnast. 1969; March, 30.

20. Hay JG. The biomechanics of sports technique, third edition. New Jersey: Prentice Hall; 1985.

21. Readhead L. Men's gymnastics coaching manual. Huddersfield: Springfield Books; 1987.

22. Fukushima S, Russell W. Men's gymnastics. London: Faber \& Faber; 1980.

23. Wilson C, Simpson SE, van Emmerik RA, Hamill J. Coordination variability and skill development in expert triple jumpers. Sports Biomech. 2008; 7:2-9.

24. Newell KM, van Emmerik RAE, Sprague RL. Stereotypy and variability. In Newell KM and Corcos DM, eds. Variability and Motor Control. Champaign, IL: Human Kinetics; 1993; 475-496.

25. Chow JY, Davids K, Button C, Rein R. Dynamics of movement patterning in learning a multiarticular action. Motor Control. 2008; $12: 219-240$.

26. Fitts PM, Posner MI. Human performance. Belmont: Brookes-Cole; 1967.

27. Harris CM, Wolpert DM. Signal-dependent noise determines motor planning. Nature. 1998; 95:780-784. 